\title{
Principles of the data reduction and first results of the fastscanning method for (sub)millimeter astronomy
}

\author{
B. Weferling, L. A. Reichertz, J. Schmid-Burgk, and E. Kreysa
}

\author{
Max-Planck-Institut für Radioastronomie, Auf dem Hügel 69, 53121 Bonn, Germany \\ e-mail: reichertz@mpifr-bonn.mpg.de; p223jsb@mpifr-bonn.mpg.de; ekreysa@mpifr-bonn.mpg.de
}

Received 22 June 2001 / Accepted 7 November 2001

\begin{abstract}
Ground-based astronomical continuum observations at (sub)millimeter wavelengths are especially difficult, because the emission of the atmospheric water vapor is usually much stronger than any astronomical signal. We present here the principles of the data reduction and first results of a new observing method that accounts for the atmospheric emission by measuring its total power with an array of detectors. We call the method fastscanning. We outline the data reduction for (sub)millimeter bolometer arrays and demonstrate the effectiveness by presenting results of astronomical observations done with the new method.
\end{abstract}

Key words. methods: observational - instrumentation: detectors - methods: data analysis - atmospheric effects

\section{Introduction}

The (sub)millimeter range is an especially difficult wave band for ground based astronomical continuum observations. Not only does the atmosphere disturb the wavefront of any electro-magnetic signal of extraterrestrial origin, as it does in most other wave-bands, but the atmosphere itself is a source of (sub)millimeter radiation much stronger than any astronomical source. This radiation comes mainly from the water vapor contained in the atmosphere. The problem can be reduced somewhat by observing from high altitudes in dry areas, but can not be avoided altogether. Things are even worse because the contribution of the atmosphere is not at all steady, but behaves in a chaotic way typical for atmospheric phenomena. The atmospheric emission is, therefore, difficult to simulate and nearly impossible to predict exactly. The classical way to tackle this problem of the skynoise is the so called dual-beam method of observation (e.g. Conway 1963). Instead of directly measuring a signal, one measures the difference between two points on the sky by quickly changing the direction of observation slightly, usually by wobbling the secondary mirror. Presuming the emission of the sky to be to a high degree similar in the two phases, one can detect fluxes of astronomical origin in the difference. The success of course depends on how far apart the two points are and how quickly one changes the position. The measurement in the single phases is usually obtained by integrating the raw signals of the bolometer during the phase to suppress high frequency noise. In the case that the extent of the source is larger than the

Send offprint requests to: B. Weferling,

e-mail: weferlin@mpifr-bonn.mpg.de angular distance of the two beams, a so-called restoring algorithm developed by Emerson, Klein and Haslam (hereafter EKH) (Emerson et al. 1979; Klein 1978) has to be applied. The dual-beam method together with the integration of the signal during the phases and the restoring algorithm is widely used as a standard procedure for the continuum mapping of astronomical (sub)millimeter sources, but it has the following disadvantages:

- A wobbler is not available at every telescope where continuum observations are of interest. The alternative of oscillating the telescope is usually not satisfactory because it is too slow;

- The scanning speed is severely constrained for a number of reasons. If the angle between the two beams becomes too large, the difference in the atmospheric emission in the two phases can no longer be neglected. Furthermore, the signal-to-noise ratio gets worse when the raw signal in the phases is integrated while moving the telescope faster;

- Usually the wobbler mechanics are restricting the scanning pattern in mapping observations, as is the case when the EKH-algorithm is used;

- It is difficult to implement the position switching in an optimal way, because of the moving parts of the mechanics which are usually quite heavy. Irregularities in the wobbling speed and wobbler-throw cause known artefacts when the restore algorithm is used (Klein 1978). They are also a source of microphonics which increases the receiver noise of the bolometers (i.e. Reichertz 1994);

- These irregularities define a small bandpass centered around the frequency of the wobbler. Components of the 
skynoise that match this bandpass, are not removed by the wobbler and lead to an increased contribution of the skynoise.

There are a number of additional problems (see Weferling 2001). The constraints of the mapping velocity are especially serious, because it is very difficult to map a fairly large part of the sky to a satisfying noise threshold in a reasonable amount of time, a task which is quite often demanded in submillimeter astronomy. One prominent example is the mapping of deep fields to detect highly redshifted (sub)millimeter sources which could indicate the development of structure in the Universe and its star forming history (see e.g. Guiderdoni 1998). Another example is the mapping of extended emission like the galactic center for example (e.g. Pierce-Price et al. 2000).

We present here a new method for astronomical (sub)millimeter continuum observations, the data reduction and first results. It works without a wobbler and avoids therefore many of the difficulties of the dual-beammethod. The method poses no limitations on the scanning velocity and the scanning pattern of a map. We call the method Fastscanning. The observational technique of Fastscanning is described in Reichertz et al. (2001). Here we develop the principles and features of fastscanning with major respect to the data analysis (Sect. 2) and present a number of millimeter maps of astronomical sources mapped with the new method (Sect. 3). We present the future perspectives and summarize the conclusions in Sect. 4.

\section{Principles and features of fastscanning}

Instead of sequentially measuring the emission of the sky with an offset from the source, one uses a second receiver simultaneously pointing slightly off-source, to measure the sky only. Then one computes the difference between the two signals to find the part which is of astronomical origin. In addition one modulates the signals in each of the receivers in a way that allows to make a distinction between the signals that are of astronomical origin and the system noise. Obviously the method will work better with a whole array of receivers, all looking at slightly different positions, because one can sample more information about the atmospheric and astronomical emission and the noise contribution of the receivers, which together make up the informational content of each signal. Arrays are common in today's (sub)millimeter observations, but the technical difficulty in making the method work is to build the single receivers in the array in a way that their behavior can be compared. There has to be a correlation between the signals of the single receivers if they are fed with the same input. Furthermore, there has to be a certain degree of correlation in the emission of the sky across the array. If the correlation is too small, the method would not work at all. We found experimentally that in modern arrays like the MAMBO system (Kreysa et al. 1999), the degree

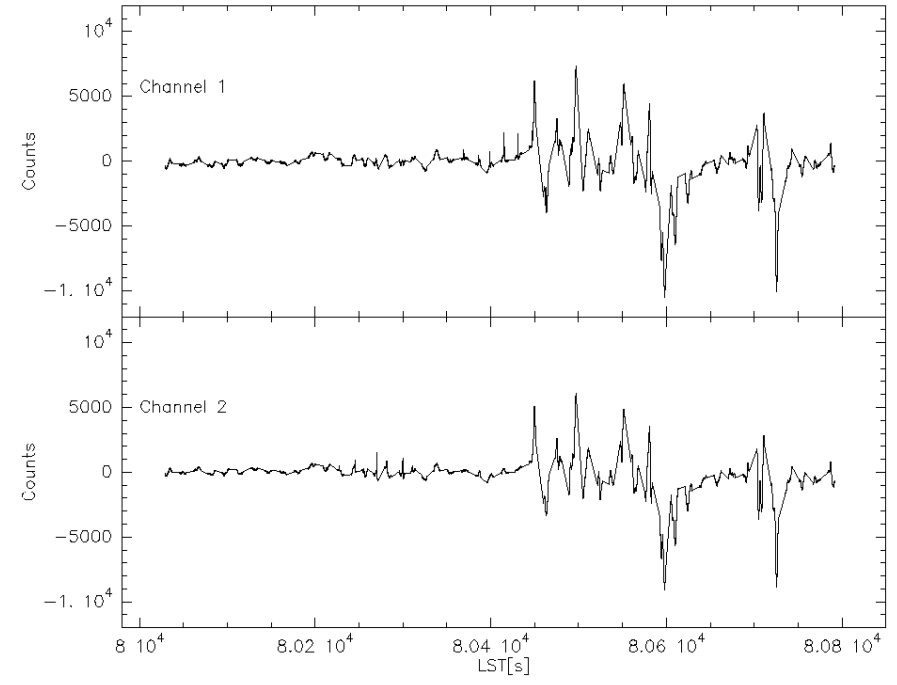

Fig. 1. A raw fastscanned signal received simultaneously from channels number 1 (top) and 2 (bottom) of the MAMBO system (Kreysa et al. 1999) during a scan of Uranus with strong skynoise during the second half of the scan. Their signals are very similar. The degree of correlation depends on the weather conditions (see Weferling 2001). The emission of the planet is dominated by the skynoise. Plotted is Counts versus Local Sidereal Time in seconds. A constant baseline was subtracted from the raw signals and a constant gain factor was applied for each channel.

of correlation between any two channels is sufficient, as Fig. 1 illustrates.

\subsection{System dependent implementation}

The implementation of the fastscanning method depends onto the telescope system which one uses for observations. We worked with the 30-m-telescope at the Pico Veleta in Spain. The following is therefore focused on this special implementation of fastscanning, but should be easily adaptable for every other millimeter-telescope. For every (sub)millimeter telescope-receiver-system it is true, that without the double beam method the signal of interest is no longer modulated at the wobbler frequency, and that the $1 / \mathrm{f}$-noise of the electronics of modern arrays is high at low (wobbling) frequencies. To still make a distinction between the noise and the signal, one has to modulate the signal into frequencies of above $1 \mathrm{~Hz}$ at least. See Reichertz et al. (2001) for a more detailed discussion. At the 30-mtelescope we generated these high frequencies by moving the telescope quickly, thus converting the spatial frequencies of a source, astronomical or atmospheric, to frequencies above the necessary threshold. The sampling rate has to be sufficiently high to match the resulting frequencies of these velocities. But it can be done otherwise.

At the JCMT, for example, the secondary mirror can be moved very quick between a variety of positions in a predefined sequence, which is called jiggling (see i.e. Cunningham \& Gear 1990). This feature is used for example in the real-time acquisition mode for SCUBA, 
DREAM (see Le Poole \& van Someren Greve 1998). DREAM is based on making the jiggling function into sky suppression modulation at the same time and depends on the supposition that the atmospheric emission does not change during a modulation cycle. It is therefore related to the concept of fastscanning.

From importance for the implementation of fastscanning is, first, that the system is capable of generating a signal in a receiver which is not disturbed by the receiver noise. By which kind of device this is done is not from special importance. The way we will describe in the following is, that the signal is modulated by a direct movement of the telescope. This can easily be adapted with other telescopes because there is no need for further hardware. Second: an array of receivers is used, to analyze the content of the atmospheric emission. It is not possible to distinct the astronomical part of a signal of a single receiver from the astronomical part by means of modulation alone, as is the case for the system noise. This is because the system noise is carried by fixed and known frequencies, which is not the case for the atmospheric contribution. This is the more so because it is not possible to modulate the astronomical signal above the noise threshold without modulating the atmospheric signal at the same time. The data reduction should in any case be addressed as a system dependent problem.

\subsection{Observational parameters for the modulation of the signal with a fast moving telescope}

We assume that the received signal is modulated by fast scans of the telescope, but the discussion can easily be adapted if the modulation is done otherwise. We then define $f_{\text {sky,max }}$ as the "highest" frequency resulting from the fastscanned emission of the sky and $f_{\text {source, max }}$ as the maximum frequency produced by fast sweeping over the source. The frequency at which the source itself is variable $f_{\text {source,var }}$ can usually be neglected. We assume this for the rest of this work. These frequencies depend in practice on the noise level of the system, but this can be neglected here. $f_{\text {sky,max }}$ and $f_{\text {source,max }}$ are functions of the scanning velocity $v . f_{\text {source,max }}$ is inversely proportional to the size of the beam, which can be represented by the full width half maximum $(F W H M)$, the size of the source $W_{\text {source }}$, and proportional to the scanning velocity:

$$
f_{\text {source } \max } \sim \frac{v}{F W H M \cdot W_{\text {source }}}
$$

The dependence on the scanning velocity has to be considered when choosing the sampling rate. $f_{\text {source, max }}$ is maximal for a point source. $f_{\text {sky,max }}$ depends not only on the scanning velocity, but also on the size, velocity, temperature and height of the emitting atmospheric structures. These parameters are not well known but a typical spectrum of the emission can be measured with fastcanning. We find that the bulk of the power is characteristically well below $20 \mathrm{~Hz}$ when scanning with the 30-m-telescope with $40 \mathrm{arcsec} / \mathrm{s}$ (see Fig. 2).

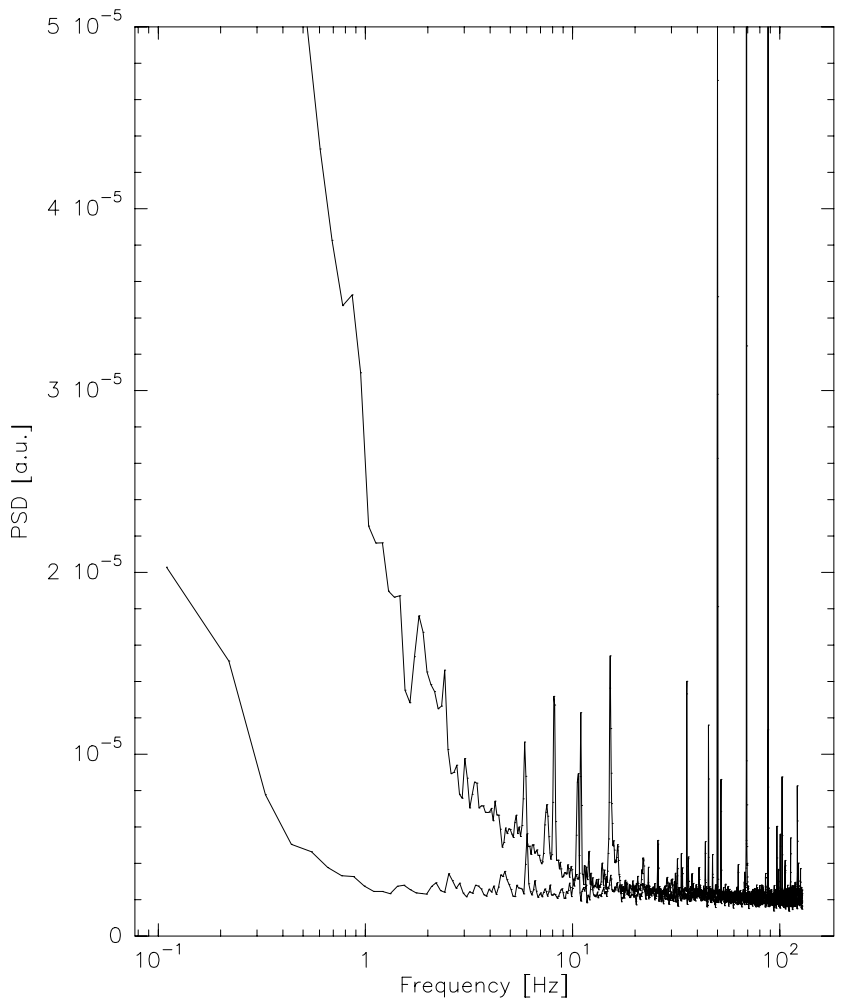

Fig. 2. The power spectrum of the sky (upper line) compared to that of the system noise (lower line). The skynoise was measured with a sampling rate of $256 \mathrm{~Hz}$ with the central receiver of the MAMBO-Array (Kreysa et al. 1999) while scanning the sky with the 30-m-telescope at Pico Veleta with a velocity of $40^{\prime \prime} / \mathrm{s}$. The system noise was measured accordingly on a $300 \mathrm{~K}$ absorber under the same conditions. Both signals were properly deconvolved with the systems filter function (see Reichertz et al. 2001). The small lines are harmonics of the DC power supply frequency.

At the 30-m-telescope we had a beam with $F W H M=$ 10.5 arcsec and a standard scanning velocity $v=40$ arc$\mathrm{sec} / \mathrm{s}$. We find that usually

$f_{\text {sky, } \max } \geq f_{\text {source,max }}$.

According to the Nyquist theorem the sampling rate has to be at least twice the maximum frequency of the signal of either astronomical or atmospheric origin. This is also the case when the signal is modulated otherwise, for example a jiggling secondary mirror.

By sampling the signal of receiver $r$ one gets a discrete function in time of the bolometer signal $S_{\mathrm{f}}(r, t)$. (We will sometimes refer to $S_{\mathrm{f}}(r, t)$ as the whole function of the signal, sometimes as the specific value of that function. The meaning can be deduced from the context.) The index "f" indicates that the signal has passed through a filter (see Reichertz et al. 2001). We assume that the measurement be taken with a constant frequency $f_{\mathrm{S}}$, so that $S_{\mathrm{f}}(r, t)$ is defined at $t_{1}, t_{2}, \ldots, t_{\mathrm{N}}$, where the total number of measurements depends on the total duration $T$ of the scan. As is described in Reichertz et al. (2001) the signal gets filtered. This can be expressed mathematically by a multiplication with the filter-function and can be reversed by 
a division accordingly. The filtering results in a loss in the efficiency which has to be considered when choosing the optimal observing strategy.

\subsection{The data reduction}

We now try to outline the principles we applied in the data reduction to achieve the results presented in Sect. 3. Because of the unique nature of the skynoise in the (sub)millimeter range and because with the dual-beammethod this problem is largely tackled for each bolometer independently, a general theory for the data reduction of bolometer arrays is missing. One has to solve the problem of the atmospheric (sub)millimeter emission in a way that can be adapted for the specific nature of the observations and the specific array given. The modulation of the pictorial content of the sky - by quick movements of the telescope, by a jiggling secondary mirror or otherwise defines a set of samples of the emission of the sky. The data treatment can be regarded as an estimation process of intensities on these samples, as is the case with other acquisition modes with millimeter-arrays (see i.e. Le Poole \& van Someren Greve 1998). The instantaneous readings of the bolometers must be modeled as caused by different components. The specific model is to be formulated to match the system of observation. The task of the reduction then is to estimate the model parameters from the data. The raw signals are usually dominated by the atmospheric emission and noise and one is interested in the part of the signal which is of astronomical origin. The atmosphere is a chaotic physical system. Its (sub)millimeter emission can, therefore, not be predicted precisely, but only approximated. The task is to estimate the different intensities from the set of measurements. We will now describe the basic principles of the data reduction that we have applied to our data to obtain the results presented in Sect. 3. We try to formulate it in a way easily adaptable for other ways of implementation of fastscanning.

By scanning (or otherwise modulating) the emission of the sky with an array and sampling the total power with a sampling rate of $f_{\mathrm{S}}$ for a total time $T$ one gets

$N_{\text {tot }}=T \cdot f_{\mathrm{S}} \cdot R=N_{\text {rec }} \cdot R$

measurements, $R$ being the number of receivers in the array and $N_{\text {rec }}$ the number of measurements (dumps) per receiver. The measurements are assumed to be taken with a constant $f_{\mathrm{S}}$, so we have a measurement at $t_{1}, t_{2}, \ldots$, $t_{N_{\text {rec }}}$ with

$t_{i+1}-t_{i}=\frac{1}{f_{\mathrm{S}}}$

We assume these signals to be deconvolved with the filterfunction.

\subsubsection{The atmospheric function}

The signal $S(r, t)$ of each receiver $r$ can at any given moment of measurement $t$ be expressed as the sum of differ- ent components. The following expression, based on the concept of statistical dependency, is fruitful for further analysis:

$$
S(r, t)=S_{Q}(r, t)+S_{\mathrm{atm}}(r, t)+\Delta_{\mathrm{atm}}(r, t)+\Delta_{\text {noise }}(r, t) \text {. }
$$

$S_{Q}(r, t)$ represents the part which has its origin in the astronomical source. We assume these sources not to be intrinsically variable during the scan. $\Delta_{\text {noise }}(r, t)$ represents the noise of the receiver. $S_{\mathrm{atm}}(r, t)+\Delta_{\mathrm{atm}}(r, t)$ represents the emission of the atmosphere. $S_{\text {atm }}(r, t)$ is statistically depended on the set of all available signals of the scan (or at all), which is represented by $M$ and is a computable function of that set:

$S_{\mathrm{atm}}(r, t)=f_{A}(M, r, t)$.

We will call $f_{A}$ the atmospheric function. $\Delta_{\text {atm }}(r, t)$ is supposed to be statistically independent from $M$ and cannot be analyzed on the basis of fundamental principles. Because we see that $\Delta_{\text {noise }}(r, t)$ is also statistically independent from $M$, whereas $S_{Q}(r, t)$ is not, we have expressed $S(r, t)$ as a sum of either statistically dependent or independent components of $M$.

If the assumption holds that

$S_{\text {atm }}(r, t) \gg\left(\Delta_{\text {atm }}(r, t)+\Delta_{\text {noise }}\right)$,

the part of the signal which is of astronomical origin can be expressed as

$S_{Q}(r, t) \approx S(r, t)-f_{A}(M, r, t)$.

If other parts of the signal are of interest, they can be found in the same way.

Equation (7) poses a demand on the construction of the array, the receiver noise has to be low and the single receivers have to be comparable in their behavior. It poses furthermore a demand on the special way of observation. The data has to be obtained in a way that information is gathered which allows a distinction between the different components of the emission. For example: the sampling rate may be to low or the distance between the samples may be to small to be essentially unrelated in astronomical content. But only the experiment can decide if Eq. (7) holds, because this is also a question of the atmospheric conditions.

Of course the atmospheric function is unknown. We will therefore use approximation functions

$f_{N}(M, r, t) \approx f_{A}(M, r, t)$,

which have an error given by

$f_{A}(M, r, t)=f_{N}(M, r, t)+\Delta f_{N}(r, t)$.

The atmospheric function is therefore the optimum of the data reduction. The approximation function we chose, represents our model of the different components which are part of each bolometer signal. The computation of a good approximation function is an estimation process of the intensities of these components. Whether or not it is possible 
to apply fastscanning for an astronomical measurement depends not only on the whether Eq. (7) holds, but also on the ability of the observer to find a good approximation to the atmospheric function. This fact expresses the fundamental principle of fastscanning, that the problem of the skynoise is not tackled by a hardware solution, like in the dual-beam-method, but by software. The data reduction is therefore much more important. In a way, the atmospheric function constitutes a software substitute for the second beam in the dual-beam method. The fundamental problem of the data reduction can be formulated in different ways. We chose the concept of the atmospheric function because within it, any specific approach can easily be formulated.

\subsubsection{Simple approximations for the atmospheric function}

Because of the very large amount of data (for example: a standard map with MAMBO at the 30-m-telescope consists of roughly 1.5 million single signals), in practice it is necessary to have only a few guiding principles which allow the construction of algorithms that can be run on a computer and which give an acceptable result. There are some well known standard reduction algorithms (see e.g. Broguiere et al. 1998), such as the baseline reduction or the application of a gain-factor, which does not suit the needs of fastscanning data, but has the advantage, of being common and sometimes more intuitively insightful. Concepts of least squares estimations are in use with comparable modes of observation such as DREAM (Le Poole \& van Someren Greve 1998). They also level out the differences in the measuring behavior of the single receivers of the array. For now we assume identical receivers, which have identical measuring behavior.

In the absence of a general theory of atmospheric (sub)millimeter emission, approximations of the astronomical emission, which means an approximation of the atmospheric function, can only be estimated by an analysis of the whole data set $M$. We applied two obvious guiding principles for the construction of simple approximation functions: first, that the structures of the atmospheric emission are connected in space and time (Principle of locality). Second, that the degree of correlation of two signals of atmospheric origin is inversely proportional to their distance in time and to the distance of the involved receivers (Principle of correlation). The second principle is obviously more demanding than the self-evident first principle. See also Klein (1978) and Weferling (2001).

The smallest possible array has two receivers, a and b. In first approximation one can make the assumption that the atmospheric emission in a is equal to the atmospheric emission in $b$ at all times (Neighbor Approximation). Because both receivers have some noise the mean of the two signals should represent a better approximation to the atmospheric emission (see e.g. Sachs 1968). For an array with $R$ receivers this can be expressed in the Mean Approximation

$f_{\bar{S}(t)}(r, t)=\bar{S}(r, t)=\frac{1}{R} \cdot \sum_{r^{\prime}=1}^{R} S\left(r^{\prime}, t\right) \quad \forall t$.

If there is no more further information, the Mean Approximation is the best approximation to the atmospheric function, which just includes signals taken at the same point in time. Further analysis may show that it is better to exclude some channels from the mean. They may be defect for example, or include an unwanted spike. The Principle of correlation suggests, that receivers close to one-another "see" similar atmospheric emission. In the Vicinity Approximation the mean is just taken over a subset of receivers:

$f_{U(r, x)}(M, r, t)=\bar{S}_{r, x}\left(U_{r, x}, t\right)=\frac{1}{x} \cdot \sum_{\substack{r^{\prime}=1 \\ r^{\prime} \subset U_{r, x}}}^{x} S\left(r^{\prime}, t\right) \forall t$

where the vicinity $U_{r, x}$ is a subset of $x$ receivers of the array. We define $\dot{U}_{r, x}$ as the same vicinity excluding receiver $r$ or another explicitly given channel, and $U^{N}(r, x)$ as the vicinity of the neighborhood of $r$, composed of the $x$ receivers closest to $r$. Neither definition is explicit, but this does not change the results to a first order.

The simple Vicinity Approximation can be improved by weighting the receivers' signals in the mean (Weighted Vicinity Approximation)

$f_{U_{W}(r, x)}(M, r, t)=\frac{1}{x} \cdot \sum_{\substack{r^{\prime} 1 \\ r^{\prime} \subset U_{r, x}}}^{x} a\left(r^{\prime}, r, t\right) \cdot S\left(r^{\prime}, t\right)$

The weighting factors $a\left(r^{\prime}, r, t\right)$ can be derived from any conceivable information, e.g. an analysis of the correlation between the receivers.

\subsection{Error of approximation}

Greater vicinitys are better for statistical reasons (see Eq. (18)). However, this involves the danger of including channels with signals consisting of very different atmospheric emission or even emission of astronomical origin from a source previously unknown. In that case it would be better to leave this channel out of the vicinity subset, which can be expressed by using $\dot{U}_{r, x}$ instead of $U_{r, x}$. In an iterated reduction it may be possible to improve the vicinity this way. One can calculate the error when using $U_{r, x}$ instead of $\dot{U}_{r, x}$, in the Vicinity Approximation. It can be computed the same way for other approximation functions:

$\Delta f_{U\left(r_{i}, x\right)}\left(r_{i}, t\right)=f_{U\left(r_{i}, x\right)}\left(M, r_{i}, t\right)-f_{\dot{U}\left(r_{i}, x\right)}\left(M, r_{i}, t\right) .(14)$

It follows that:

$\Delta f_{U\left(r_{i}, x\right)}\left(r_{i}, t\right)=\bar{S}_{r_{i}, x}\left(U_{r_{i}, x}, t\right)-\bar{S}_{r_{i}, x}\left(\dot{U}_{r_{i}, x}, t\right)$ 
and

$$
\begin{aligned}
\Delta f_{U\left(r_{i}, x\right)}\left(r_{i}, t\right)=\frac{1}{x} \cdot \sum_{\substack{r^{\prime}=1 \\
r^{\prime} \subset U_{r, x}}}^{x} S\left(r^{\prime}, t\right) \\
-\frac{1}{x-1} \cdot \sum_{\substack{r^{\prime}=1 \\
r^{\prime} \subset U_{r, x}, r^{\prime} \neq r_{i}}}^{x} S\left(r^{\prime}, t\right) .
\end{aligned}
$$

Which gives

$$
\Delta f_{U\left(r_{i}, x\right)}\left(r_{i}, t\right)=\frac{S\left(r_{i}, t\right)}{x}+\frac{\sum_{\substack{r^{\prime}=1 \\ r^{\prime} \subset U_{r, x}, r^{\prime} \neq r_{i}}}^{x} S\left(r^{\prime}, t\right)}{\sum_{\substack{r^{\prime}=1 \\ r^{\prime} \subset U_{r, x}, r^{\prime} \neq r_{i}}}^{x} S\left(r^{\prime}, t\right)}
$$

and we find the source-error to be

$\Delta f_{U(r, x)}(r, t)=\frac{S_{Q}(r, t)}{x}-\frac{f_{\dot{U}}(r, x)(M, r, t)}{x}$

or accordingly, if another component carries the error than $S_{Q}\left(r_{i}, t\right)$. In the last equation the index $i$ was dropped, because the equation holds for either receiver. The result can easily be generalized if the Weighted Vicinity Approximation is used.

If $R \gg 1$ then the source-error can be approximated by

$\Delta f_{U(r, x)}(r, t) \approx \frac{S_{Q}(r, t)}{x}$,

favoring great vicinities. Equation (17) demonstrates the importance that any given information about the extent and position of the source should be used. Especially because the source-error adds up, if more than one receiver "sees" a source. The same is true for the noise-error. Assuming that for a given moment the atmospheric emission in all receivers is equal within the limits of white noise and assuming that this is the same for all receivers and has a standard deviation of $\sigma_{\text {noise }}$, the noise-error is

$\Delta f_{U(r, x), \text { noise }}(r, t) \approx \frac{\sigma_{\text {noise }}}{x}$,

also favoring great vicinities.

\subsection{The atmospheric function in the context of classical data reduction}

One can formulate the approach of the Weighted Approximation Function in a way that takes into account some classical means of data reduction:

$$
\begin{aligned}
& f_{U_{W}(r, x)}(M, r, t)=\frac{1}{x} \cdot \sum_{r^{\prime}=1}^{x} a\left(r^{\prime}, r, t\right) \cdot S_{\mathrm{c}}\left(r^{\prime}, t\right) \\
& =\frac{1}{x} \cdot \sum_{r^{\prime}=1}^{x} a\left(r^{\prime}, r, t\right) \cdot\left(S\left(r^{\prime}, t\right) \cdot g_{B}\left(r^{\prime}, t\right)-b\left(r^{\prime}, t\right)\right)
\end{aligned}
$$

The pre-corrected signal $S_{\mathrm{c}}\left(r^{\prime}, t\right)$ results from the application of a time dependent gain-factor and a baseline. Both can be fitted to the data to reduce differences in the behavior of the receivers (see Weferling 2001).

\subsubsection{Time dependent approaches}

All the previous approximation functions just use signals taken at the same time as the signal which is to be corrected, which is in accordance with the principle of correlation. There are two situations which make it necessary to deviate from that principle. First, one may detect a specific atmospheric structure in the signals by an analysis of the data. Second, some receivers see the emission of an astronomical source at a given time. Both cases may be deduced from analysis or other means. The second one is easier to handle because the emission from an astronomical object can be assumed to be stationary at the sky. This is not the case for atmospherical emission. Its is therefore important for the data reduction to take into account the specific mode of observation. One must take into account the direction dependence of the telluric sky with viewing direction (for further details see Weferling 2001). The problem for data reduction is therefore in this sense defined by the pattern of observation on the sky, which is defined by the capabilities of the telescope system (i.e. the presence of a jiggling secondary) and can be optimized for the specific observational task within the requirements of the fastscanning method. Including inappropriate receivers into one form of the Vicinity Approximation increases the source-error according to Eq. (17). Especially with extended sources the accumulated error may become too large. As a last resort one can chose the signals which have a greater distance (in time) from the signal to be corrected but can be presumed to be clear of any relevant astronomical signals. Obviously, in that case, one loses similarity between the mixed signals. One has to find a way of reaching a good deal in this trade-off.

There are a huge number of possibilities to chose a subset of signals from $M$, even for a small array and a short scan. The problem is to find the subset $Q \in \bar{M}$ (where $\bar{M}$ is the set consisting of all subsets from $M$ with at least one element) and weighting parameters accordingly which minimize the error in the General Weighted Vicinity Approximation

$f_{Q}=\frac{1}{m(Q)}(M, r, t) \sum_{S\left(r^{\prime}, t^{\prime}\right) \in Q} a\left(r^{\prime}, r, t^{\prime}, t\right) S\left(r^{\prime}, t^{\prime}\right)$,

and in this way solve an extreme--values-problem:

$\Delta f_{Q}(M, r, t) \leq \Delta f_{X}(M, r, t) \quad \forall X \subset \bar{M}$.

Because the atmospheric function is generally unknown one has to resort to methods which are generally known from comparable problems from estimation theory. The authors are currently working on that. 


\subsubsection{Summary}

There is no general solution for the data reduction. The optimal way of reduction always depends on the set of data, the observational goal and the telescope-receiversystem. The considerations put forth so far, have to be adapted to each specific case. It should, thereby, be possible to find solutions to the problem of the atmospheric emission, that go beyond the given approximations, within the framework given here. Especially the development of an algorithm for finding a solution for Eq. (19) should be given some thought. The authors are working on that.

For practical reasons it is necessary to have a robust solution for the atmospherical problem, which can be applied by every observer without giving the reduction too much thought. We, therefore, now apply the fairly simple Vicinity Approximation together with a pre-corrected signal, by applying a baseline and a gain. This comes as close to the common standard reduction as possible for fastscanning data and works well with the receiver we have used. We thereby examine the robustness of this approach and demonstrate that fastscanning is a useful tool for many projects in (sub)millimeter astronomy. Further studies can be developed on that basis.

\section{First fastscanning results}

\subsection{General}

We present here a number of results of observations with the fastscanning method done with the 30-m-telescope at Pico Veleta, Spain and compare them to results of the dual-beam-method.

We used the MAMBO bolometer array with 37 channels (35 operating) (Kreysa et al. 1999) operating at $250 \mathrm{GHz}$ together with a backend capable of monitoring the response of up to 128 receivers with a sufficiently high sampling rate (see Reichertz et al. 2001). In fastscanning mode we used a scanning velocity of $v=40 \mathrm{arcsec} / \mathrm{s}$ and a sampling rate of $f_{S}=125 \mathrm{~Hz}$. In the case we did not perform a dual-beam-map for direct comparison, we were able to compare the results with many maps of a wide range of astronomical sources made with MAMBO at the 30-m during Winter 1999/2000.

Maps of both styles have been reduced in the most similar way possible, so that neither method has been given extra care. We used the Vicinity Approximation from Eq. (12) with the pre-corrected signal to reduce the fastscanning data.

We were able to produce roughly 50 fastscanned maps of a large variety of objects. We produced many of these maps with the extension of $240^{\prime \prime} \times 200^{\prime \prime}$, which is a standard size for dual-beam maps, for better comparison. One common way to measure the quality of a map is the residual noise. Table 1 shows the results of the direct comparison of the double-beam method with fastscanning in terms of residual noise and integration time for a standard map. The double-beam maps have been taken in the so called on-the-fly mapping mode, in which the telescope is scanning the sky while the secondary mirror is chopping at the same time. The residual noise is measured by the rms computed for each map with a set of polygons in the central region. In this way the whole map is regarded as the target, so that both, the On-beam as well as the Off-beam contribute to the integration time, given in column three of Table 1. This contrasts to the way in which the simple On-Off mode of observation is usually regarded.

Due to the scanning speed, fastscanning takes just $306 \mathrm{~s}$ of integration time for a standard map of $240^{\prime \prime} \times 200^{\prime \prime}$. The noise level reachable in $3060 \mathrm{~s}$, the integration time of a dual-beam map of standard size, is given by

$\mathrm{rms}_{3060 \mathrm{~s}} \approx \frac{23.0}{\sqrt{3060 / 306}}=7.3 \mathrm{mJy}$.

This equals fairly good the residual noise of the on-the-fly double-beam maps. It is also a direct result of the map in Fig. 5b.

The net result is, that within the margin of error, one reaches the same quality in the maps in terms of residual noise with the relatively simple approach of the Vicinity Approximation in the same time of integration. The margins of error are relatively large for fastscanning, because, due to bad weather during all observations, the calibration of the data has a relative margin of error of about $15 \%$, which holds for all given fastscanning fluxes given in this paper. In the Vicinity Approximation the known bad receivers and receivers which could be presumed to see strong astronomical emission were left out. No further iteration was done.

During our tests it turned out, that with the MAMBOBolometer array there is a typical correlation between the signals of about $90 \ldots 95$ percent in intervals of $5 \mathrm{~s}$, which on first approximation is constant across the whole array, thus explaining the effectiveness of the Vicinity Approximation. On first approximation, the emission of the sky was the same across the whole array, but this depended on the weather conditions. In some weather conditions we were also able to observe differences in the degree of correlation depending on the relative positions of the receivers, confirming the assumption of the Principle of correlation. A further, more sophisticated data reduction, can thus be expected to improve the residual noise. The authors are currently working on this.

The size of a standard map is very unfortunate for fastscanning, because there is a large overhead due to the programmed scanning pattern of the 30-m-telescope in mapping mode (compare columns two and three in Table 1), which has an overhead period of approximately $5 \mathrm{~s}$ at the end of each subscan, nearly matching the integration time of $6 \mathrm{~s}$ per subscan. The overhead is artificially large to allow computers to process the data. It can be reduced drastically to approximately $0.3 \mathrm{~s}$ per turn (see Schraml). The typical size of a fastscanning map should therefore be much larger than that of a dual-beam map. 
Table 1. Comparison between fastscanning and the double beam method for a standard map size $240^{\prime \prime} \times 200^{\prime \prime}$. The fastscanning was done with $v=40^{\prime \prime} / \mathrm{s}$, the double beam mapping with $4^{\prime \prime} / \mathrm{s}$. Subscan spacing was $4^{\prime \prime}$ in both cases. The results in the last column are averaged over all available maps. All fastscanning maps were reduced with the Vicinity Approximation together with a pre-corrected signal. The dual-beam maps accordingly without an approximation to the atmospheric function. See also text.

\begin{tabular}{|c|c|c|c|}
\hline Method & Total Time & Integration Time & rms \\
\hline double-beam & $55.25 \mathrm{~min}$ & $3060 \mathrm{~s}$ & $7.5 \pm 1.0 \mathrm{mJy}$ \\
\hline fastscanning & $9 \mathrm{~min}$ & $306 \mathrm{~s}$ & $23.0 \pm 4.0 \mathrm{mJy}$ \\
\hline
\end{tabular}

Furthermore, with fastscanning there is no need at all to map in parallel subscans at constant elevation, as is done in a standard on-the-fly map. Instead, one is free to choose any pattern. This is because a) the wobbler implies no restrictions, b) there is no need to take two beams at the same elevation and c) no restrictions are implied by the EKH-algorim, which demands the scanning direction to be parallel to the vector between the two beams. This allows choosing a scanning pattern where the overhead is reduced, or even not present at all, taking data continuously.

\subsection{Point-sources}

Figures 3,5 and 6 show some fastscanning maps in direct comparison with dual-beam maps of the same sources. Due to the finite beam at the $30-\mathrm{m}$ telescope, sources with size much smaller than the beam size of $\sim 10.5^{\prime \prime}$ have point-like appearance.

We found no difficulties at all with the mapping of these point-sources. With MAMBO a point-source is almost never present in all receivers. In fact, apart from planets and other sources of comparable flux, the emission of a point-source is usually strong only in one receiver and negligible in all others. The source-error is thus usually small and the subset $U_{r, x}$ used in the vicinity approximation can consist of all, or all but one receiver. With strong sources, or sources of known position, the receiver "seeing" the source can be excluded. Otherwise, it can be excluded in a second step, after a first reduction has shown the source. This can be expected, for all but the faintest sources, because Eq. (17) gives a small source-error for $x=37$. The faintest sources disappear in the noise-error.

Figure 4a shows a dual-beam map of Mars, which took $52.25 \mathrm{~min}$. Beside the planet the refraction pattern of the supporting structure of the subreflector is visible and very faint compared to the emission of the planet. In Fig. 4b, a fastscanning map of Mars, not only the planet is clearly visible, but also that same supporting structure, even though the map took just 9 min and has an integration time of one-tenth of the dual-beam map. In the dualbeam map the typical artefacts of the EKH-algorithm were visible, resulting from variations in the wobblerthrow (see Klein 1978). This typical mapping error does of course not show up in the fastscanning map.

The observation of strong point-sources can therefore easily be done with fastscanning.
Figure 5 shows two fastscanning maps of the point-like Quasar 1101+609, which has a peak flux of $\sim 85 \mathrm{mJy}$. The maps were done with an optical depth of the sky of about $\sim 0.6$, at medium elevation, thus absorbing approximately half the emission. The residual noise in a typical fastscanning standard map is $23.0 \mathrm{mJy}$. The source, therefore, is not visible in Fig. 5a, which shows one such map. In Fig. 5b a mosaic of ten such maps, with a summed integration time of $3060 \mathrm{~s}$ is shown. The residual noise of this map is $7.2 \mathrm{mJy}$, as was predicted by Eq. (21). The source is clearly visible. This demonstrates that fastscanning can easily be applied for the observation of faint point-like sources and that making mosaic fastscanning maps is possible.

\subsection{Extended sources}

Sources with extended emission pose a different problem to the data reduction (at least with MAMBO), because many receivers see different parts of the source at the same time. The source-error can thus become large, compared to the emission of the source. This is especially important for the fainter part of the emission.

Figures 5 and 6 show fastscanned maps of extended sources in comparison with dual-beam maps of the same sources. Both sources are well know regions of star formation, IRAS 05490+2658 and IRAS 05358+3543 (Beuther et al. 2001).

In both cases, the structures match well on first approximation, even though the integration time of the fastmaps is just one-tenth of the dual-beam maps (and the total time less than one-fifth). The reason the structures on the fastmaps appear narrower is a result of Eq. (9) in Reichertz et al. (2001). The most extended structures suffer the most severe losses. These losses could have been reduced by moving the telescope faster (see Eq. (9) in Reichertz et al. 2001). The size of a structure within a subscan, depends, of course, on the scanning direction. Just one scan perpendicular to the first should thus recover much of the losses. Figure 6 is a good example of that. The fastmap was scanned in the most unfortunate direction, nearly parallel to the line of maximum extension of the source, so that the losses are fairly large. Due to technical problems not connected with fastscanning, we were not able to make a second map to recover the losses. A fastscanned standard map takes much less time than a dual-beam map and there is no reason to wait until a source has rotated on the sky with respect to the array because with fastscanning one is able to map as one 

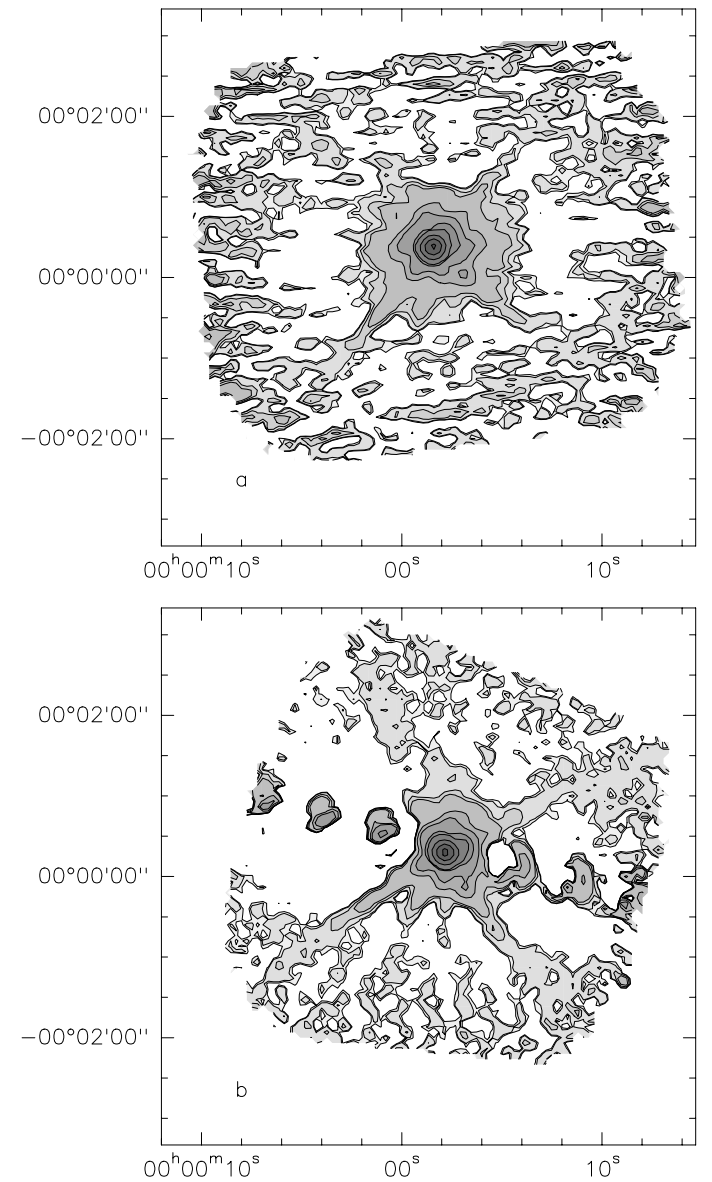

Fig. 3. b) shows a dual-beam-map of Mars, the typical artefacts of the EKH-algorithm are present; positive false images along a line to the left of the planet and negative false images to the right. a) shows a fastscanning map of Mars without these artefacts. The peak-flux is $147 \mathrm{Jy}$ in b) and 144 in a). The contours of the grey scale are at $0.001,0.01,0.05,0.5,1$, $3,5,20,50$ and $90 \%$ of the respective peak-fluxes.

pleases to. Therefore, a mosaic map in which most of the losses from the single maps are recovered, could still take less time than a dual-beam standard map. In addition, a mapping pattern where the subscans are not parallel to each other can optimize the relation between mapping time and losses (for example a $\star$-like Lissajous pattern). The authors are currently working on that. Losses are also known for the dual-beam method (Motte et al. 2001).

The maps demonstrate, that fastscanning can be a useful tool for the mapping of extended sources. The detection of strong emission can be done much faster - with smaller but still more than sufficient signal-to-noise ratio - with fastscanning than with the dual-beam method. Fastscanning can therefore be used for survey maps.

\subsection{Fastscanning with undersampling}

The specific advantages of fastscanning can be combined with other observing strategies. Sievers and Teyssier proposed a new mapping strategy (Sievers \& Teyssier 2000). In this mode, only a subset of receivers in the array
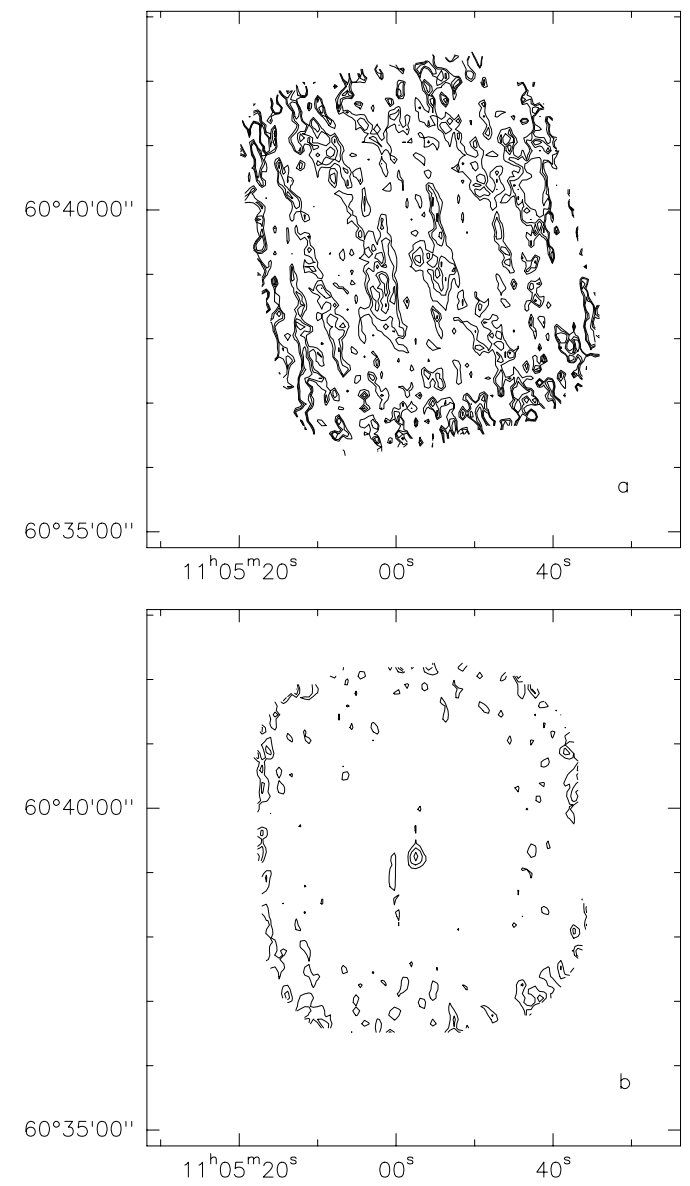

Fig. 4. The figure shows in a) a standard fastscanning map of the Quasar 1101+609 with a peak flux of $85 \mathrm{mJy}$, the source is not visible. b) Is an mosaic of ten such maps, the source is clearly visible. The noise in a) amounts to $23.0 \mathrm{mJy}$ in $\mathbf{b}$ ) to $7.2 \mathrm{mJy}$. The contours are at 74, 42 and $17 \mathrm{mJy}$ approximating roughly 90,50 and $20 \%$ of the sources peak flux. The opacity during the scans was 0.6 .

observe a given point on the sky. The observing pattern is chosen so that the observations of all receivers combined result in a map without gaps. This map, with a subscan spacing of $22^{\prime \prime}$, is undersampled compared to a normal map, which has a spacing of $4^{\prime \prime}$, therefore one loses sensitivity. One also loses resolution with this mapping strategy (Sievers \& Teyssier 2000). The amount of time in which the map can be completed is smaller in comparison to the standard mode. We have tested fastscanning in this mapping mode. The map in Fig. 7 shows the result.

The map shows the region of VLA1 HH 1-2 (i.e. Chini et al. 1997). The three main components of that source are clearly visible, although the faintest one, HH147, with a flux of approximately $111 \mathrm{mJy}$ (Chini et al. 1997) is detected with a certainty level of less than $3 \sigma$. The other two with fluxes of $160 \mathrm{mJy}$ and $450 \mathrm{mJy}$ (Chini et al. 1997) are clearly detected. No other sources are detected with a certainty level of at least $3 \sigma$. The structures at the $2.4 \sigma$ level are probably noise, besides the detection at the known position of $\mathrm{HH} 147$. The residual noise of the map is higher than that of a fastscanning map due 

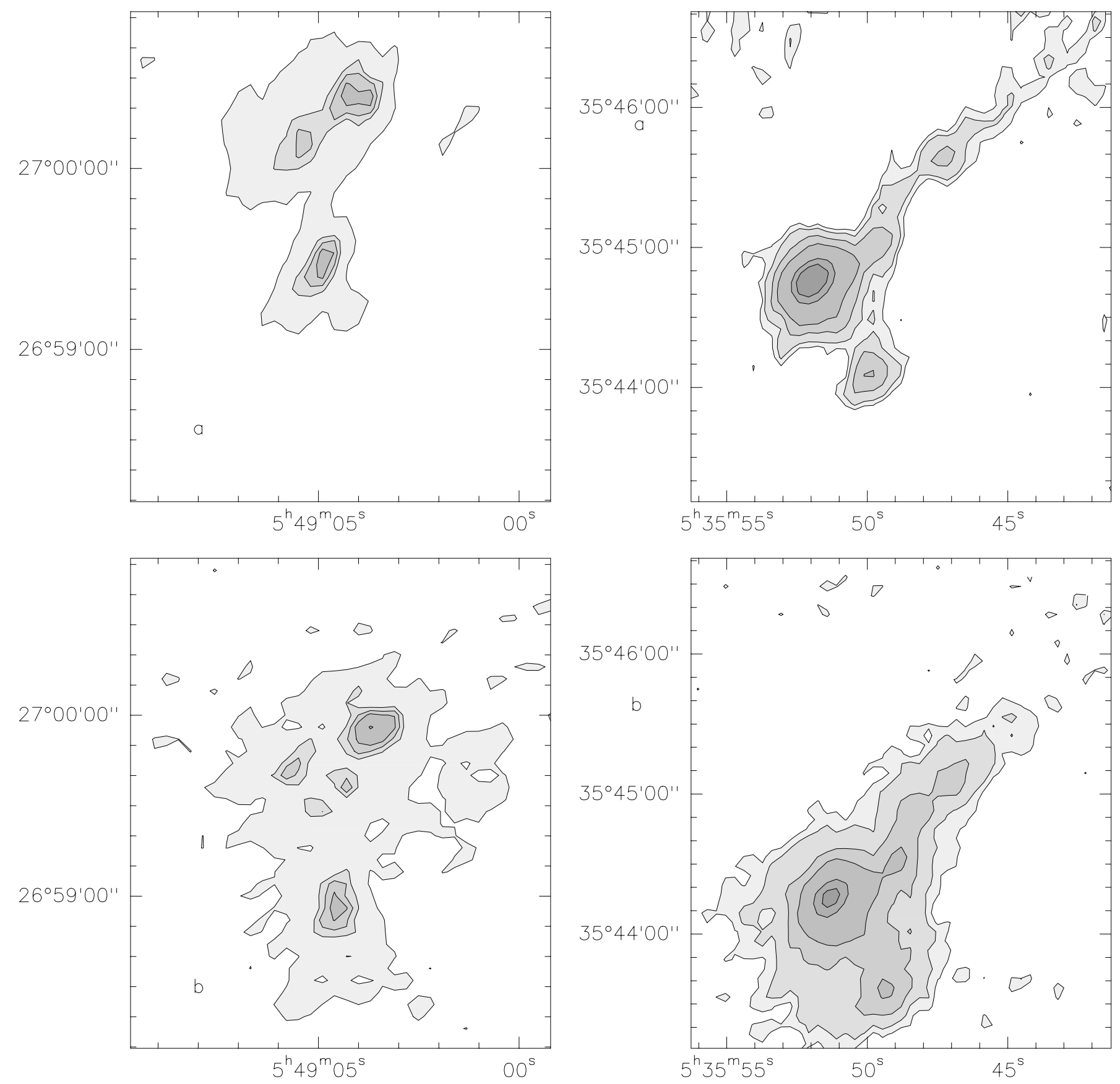

Fig. 5. a) shows part of a mosaic of two fastscanning maps of the star-forming region $05490+2658$ with a peak flux of $270 \mathrm{mJy}$. The noise of the map is $16 \mathrm{mJy}$. For comparison b gives a standard dual-beam map of the region from Beuther 2001. The contours are at 70, 150, 175, 200 and 250 mJy. Map a took in whole $18 \mathrm{~min}, \mathbf{b})$ took $55.25 \mathrm{~min}$.

to undersampling, but still low enough to detect sources of about $150 \mathrm{mJy}$ with more that $3 \sigma$. At the edge of the map the residual noise gets higher because even less receivers have observed there and has been screened out outside the effectively covered area. Due to technical difficulties not connected with fastscanning, there is a large pointing error.

Fig. 6. a) shows a fastscanning map of the star-forming region $05358+3543$ with a peak flux of $1.0 \mathrm{Jy}$. The noise of the map is $16 \mathrm{mJy}$. For comparison b gives a standard dual-beam map of the region from Beuther (2001). The contours are at 40, 70, 140, 250, 700 and $950 \mathrm{mJy}$. Map a took in whole $9 \mathrm{~min}, \mathbf{b})$ took $55.25 \mathrm{~min}$.

The total map size is $1200^{\prime \prime} \times 396^{\prime \prime}$. A map that size would have taken 8.5 hours in standard double-beam observing mode and would therefore be practically impossible to complete in one scan. It still would have taken 100 min in dual-beam mode with the mapping strategy of Sievers and Teyssier. The fastscanning map in Fig. 7 took just $10.5 \mathrm{~min}$, which is just 10 per cent of the above mentioned time and just 2 per cent of the time the 


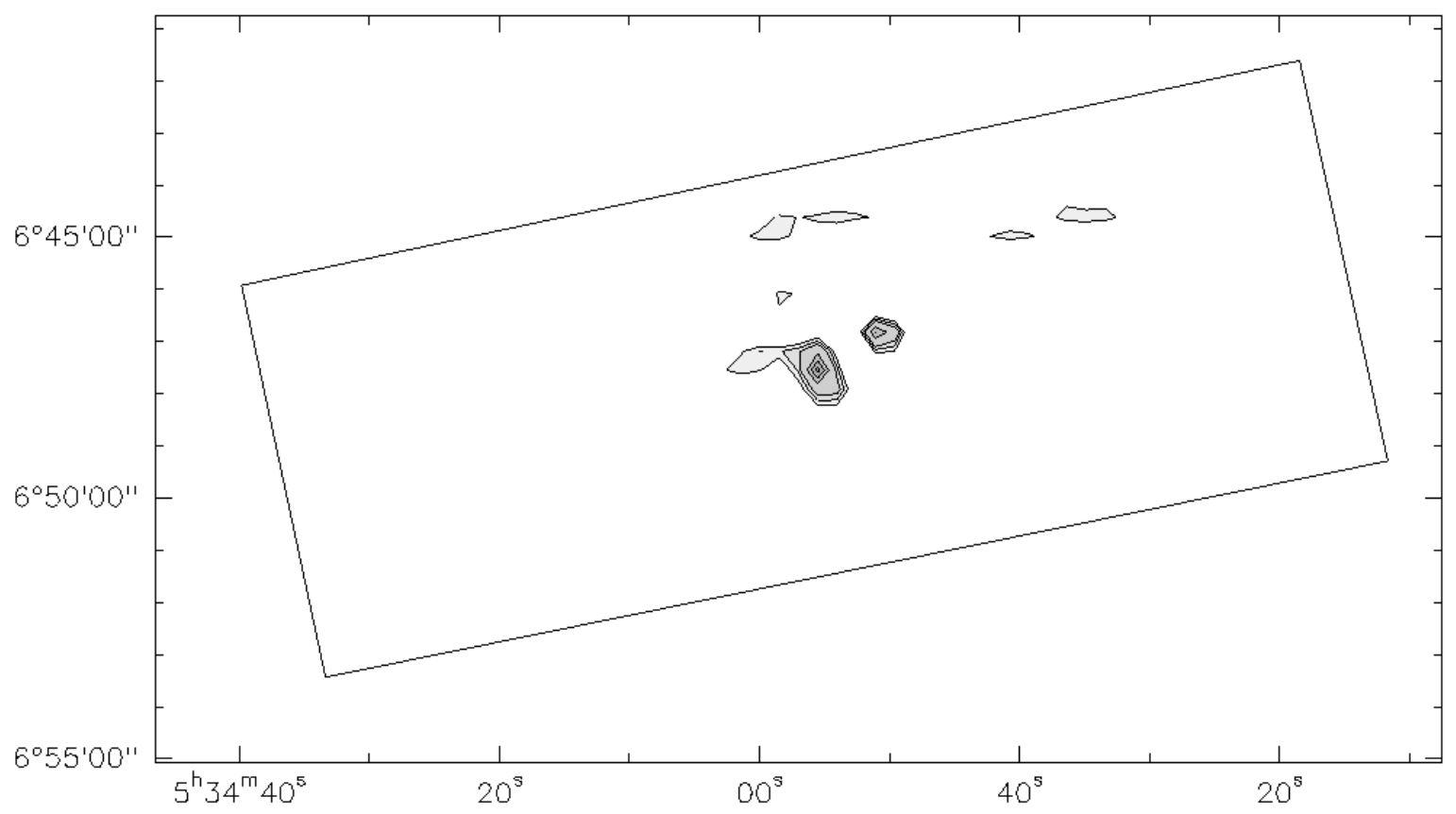

Fig. 7. A fastscanned map of HH 1-2, observed with the strategy from Sievers and Teyssier. The total extension of the map is $1200^{\prime \prime} \times 396^{\prime \prime}$ The effective map size is roughly indicated by the solid polygon. The contours represent certainty levels of $2.4,3$, $4,5,7.5$ and $12 \sigma$.

standard mode would have taken. This underlines the importance of fastscanning as a tool to reduce observing time especially in surveys of relatively strong sources.

\section{Conclusions and outlook}

Fastscanning is a new method for (sub)millimeter observations which in some cases offers a variety of advantages over the double-beam technique.

It is no longer necessary to use a wobbler or a focal plane chopper or something else to generate an off-beam as long as an array of receivers is available. The problem of the skynoise is tackled by software instead of hardware. This can simplify the construction of new telescopes by relaxing the requirements of the wobbler. Especially the construction of (sub)millimeter-telescopes on hot-airballoons becomes easier when fastscanning is used. One is also able to make continuum (sub)millimeter observations at sites where no wobbling device is present. Telescopes without a wobbler, which were previously not designed to observe at (sub)millimeter wavelengths, can now be used for this purpose.

Some experiments in submillimeter astronomy can be carried out in a shorter time than what the double-beam method requires with a still sufficient signal-to-noise ratio, e.g. the mapping of strong sources, because of the increased scanning speeds possible with fastscanning. Even surveys may become feasible, especially when using the mapping mode developed by A. Sievers (see Sievers \& Teyssier 2000) together with fastscanning. All other observations can at least be made with as good a quality as that of the dual-beam method given the same time of integration. It is to be expected, that with a further devel- opment of the theory of the data reduction for arrays as well as with the development of more advanced mapping patterns, fastscanning observations could take the advantage over dual-beam observations.

The coordinate system of observation is not restricted by the mechanics of a wobbler. The observer using fastscanning is free to let the telescope scan in any pattern.

All irregularities induced by the wobbler do not matter in the case of fastscanning.

Although a fast moving telescope is the most straightforward way, the method can be implemented with other hardware systems, i.e. a jiggling secondary mirror.

The price of these advantages are computer storage space and processing power. The amount of raw data necessary to produce comparable maps is higher by a factor of roughly $f_{S}$, but the increase of the amount of raw data should by no means be a serious constraint. The only reason to worry, is when strong constraints on the data transmission rate are imposed, as can be the case with airborne observations.

The authors are currently working on optimizing fastscanning. The question of how different filter functions and observing parameters can lead to a further improvement is of special interest. Also, one might improve the data reduction by further analysis of the correlation between the receivers, something which is under study. The identification of parameters which are to be found in the data reduction and the estimation of these from the data need further consideration (see Weferling 2001, where this is described more detailed.) The development of more complex scanning patterns is also being investigated. All 
this should further expand the already huge possibilities of fastscanning.

Acknowledgements. The authors would like to thank D. Muders, F. Motte and J. Gromke for discussions as well as W. Brunswick, A. Sievers, H. Wiesemeyer. We also thank A. Karastergiou and T. Stanke. We are also grateful to R. S. Le Poole for valuable suggestions which improved this article a lot.

\section{References}

Bracewell, R. N. 1999, The Fourier Transform and its Applications (New York)

Beuther, H., Schilke, P., Menten, K., Sridharan, \& T. K., Wyrowski, F. 2001, ApJ, submitted

Broguiere, D., Neri, R., \& Sievers, A. 1998, NIC Bolometer Users Guide, Grenoble

Chini, R., Reipurth, B., Sievers, A., et al. 1997, A\&A, 325, 542

Conway, R. G., Daintree, E. J., \& Long, R. J. 1965, MNRAS, 131,159

Cunningham, C. R., \& Gear, W. K. 1990, SCUBA: submillimeter common-user bolometer array for the James Clerk Maxwell Telescope, Proc. SPIE 1235, 515

Emerson, D. T., Klein, U., \& Haslam, C. G. T. 1979, A\&A, 76,92

Guiderdoni, B., Hivon, E., Bouchet, F. R., \& Maffei, B. 1998, MNRAS, 295, 877
Klein, U. 1978, Kontinuumsbeobachtungen Ausgedehnter Radioquellen mit der Dual-Beam Methode, Diploma thesis, Bonn

Kreysa, E., Gemünd, H. P., Gromke, J., et al. 1999, Infr. Phys. Techn., 40, 191

Le Poole, R. S., \& van Someren Greve, H. W. 1998, DREAM, the Dutch REal-time Acquisition Mode for SCUBA, SPIE, 3357, 638

Motte, F., \& Andre, P. 2001, A\&A, 365, 440

Pierce-Price, D., Richer, J. S., Greaves, J. S., et al. 2000, ApJ, 545, L121

Philipp, S., \& Zylka, R. 1998, The central regions of the Galaxy and galaxies, Proceedings of the 184th symposium of the International Astronomical Union, held in Kyoto, Japan, August 18-22, 1997, ed. Yoshiaki Sofue (Dordrecht: Kluwer) 63

Reichertz, L. A. 1994, Ein 100 mK-Bolometersystem zur Detektion von $\mathrm{mm} / \mathrm{submm}-$ Strahlung, Ph.D. Thesis, Bonn

Reichertz, L. A., Weferling, B., Esch, W., \& Kreysa, E. 2001, A\&A, 379, 735

Sachs, L. 1968, Statistische Auswertungsmethoden, Heidelberg Schraml, J. 2000, personal communication

Sievers, A., \& Teyssier, D. 2000, A Fast-Mapping Method for Bolometer Arrays: Comissioning tests

Weferling, B. 2001, Fastscanning - Astronomische Beobachtungen mit einer neuen Messstrategie für (Sub-)Millimeter-Kontinuumsarrays, Ph.D. Thesis, Bonn 